\title{
Grazing-incidence iridescence from a butterfly wing
}

\author{
Chris Lawrence, Peter Vukusic, and Roy Sambles
}

\begin{abstract}
The Troides magellanus butterfly exhibits a specialized iridescence that is visible only when its hind wings are both illuminated and viewed at near-grazing incidence. The effect is due to the presence of a constrained bigrating structure in its wing scales that has been previously observed in only one other species of butterfly (Ancyluris meliboeus). However, whereas the Ancyluris presents wide-angle flickering iridescence, the Troides butterfly uses pigmentary coloration at all but a narrow tailored range of angles, producing a characteristic effect. (C) 2002 Optical Society of America

OCIS codes: $\quad 050.1950,050.1960,050.1970,170.1420$.
\end{abstract}

\section{Introduction}

Butterflies and moths (Lepidoptera) are renowned for the variety of colors and patterns exhibited by their wings, all of which derive from the scales that coat them. The scales are flat plates of $\sim 100 \mu \mathrm{m}$ in width, arranged in an overlapping roof tile pattern to a species-dependent density of $200-600$ scales $/ \mathrm{mm}^{2}$ (Fig. 1). The scales consist of cuticle, a neartransparent polymeric material, and are commonly impregnated with a chemical pigment such that each scale provides a single colored element in the overall wing pattern.

However, it is often found that a scale's appearance is dictated by its geometric structure rather than by chemical chromophores. Such scales are intricately shaped with stratification, ${ }^{1}$ voids, ${ }^{2}$ and complex groove shapes ${ }^{3}$ to harness effects such as multilayer interference, scattering, and diffraction. These structural color techniques provide high reflectivities and iridescent effects, both of which are useful techniques when butterflies signal to competitors and potential mates.

The Troides magellanus butterfly inhabits the Philippines. It is patterned with yellow and black scales, their colors deriving from chemical means:

At the time of this research, C. Lawrence was with the Defence Evaluation and Research Agency, Farnborough, Hants GU14 0LX, United Kingdom. He is now with QinetiQ, Ively Road, Farnborough, Hampshire GU14 0LX, United Kingdom and can be reached at crlawrence@qinetiq.com. P. Vukusic and R. Sambles are with the Thin Film Photonics Group, School of Physics, University of Exeter, Stocker Road, Exeter EX4 6NA, United Kingdom.

Received 28 April 2001; revised manuscript received 17 October 2001.

0003-6935/02/030437-05\$15.00/0

(C) 2002 Optical Society of America
Melanin provides the black pigment, whereas the yellow is produced by a specialized chemical chromophore known as a papiliochrome. However, under specific illumination conditions the butterfly exhibits iridescence: If the yellow section on the hind wing is both illuminated and viewed from the outermost edge at near-grazing incidence, it will produce a flash of blue-green light that is clearly visible to the naked eye.

To our knowledge, grazing-incidence iridescent effects in Lepidoptera have never been reported in the scientific literature. Furthermore, it was not immediately obvious how the wings combined strong pigmentary coloration with such a striking and angle-specific effect, and an unusual structural coloration mechanism was anticipated. In this paper we report how we managed to fully elucidate the mechanism of this unusual iridescent effect by a combination of microscopy, spectroscopy, and mathematical modeling.

\section{Experimental Results}

\section{A. Electron Microscopy}

We determined the shape and surface texture of the scales by obtaining scanning electron microscope (SEM) images from small sections of a wing (Fig. 2). Samples were sputtered with $5 \mathrm{~nm}$ of gold to provide a conducting surface and were examined with a Hitachi S-3200N electron microscope. It should be noted that previous studies ${ }^{1,3}$ have proven that there is no significant distortion of the scale geometry during this process.

Even relatively low-magnification images of the $T$. magellanus wing (Fig. 3) reveal distinct ridges that run the length of the scales, at a spacing of approximately $1200 \pm 50 \mathrm{~nm}$ and of $2200 \pm 50 \mathrm{~nm}$ in height. 


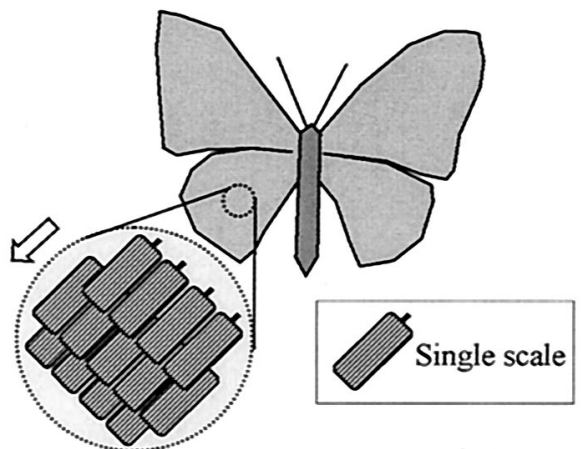

Fig. 1. Schematic of the roof tile arrangement of scales on the butterfly's wings. The arrow points toward the tip of the hind wing, running parallel to the scale ridges, and is repeated in Figs. $2-4$ and 7.

These are fairly typical of most lepidopteran scales, except for the fact that each ridge exhibits a series of platelike branches that extend to either side. These plates are tilted to an angle of $36 \mathrm{deg}$ to the vertical (Fig. 4) and hence form a multilayer stack when the scale is viewed at near-grazing incidence. As measured along the top of the ridge, the repeat period of the plates is approximately $260 \mathrm{~nm}$.

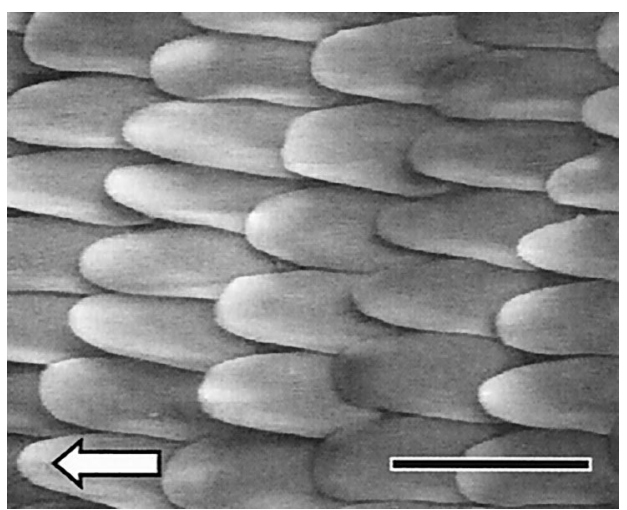

Fig. 2. SEM image of the surface of a typical butterfly wing ( $T$. magellanus), with an arrow pointing toward the tip of the hind wing (see Fig. 1). Scale bar is $\sim 150 \mu \mathrm{m}$ long.

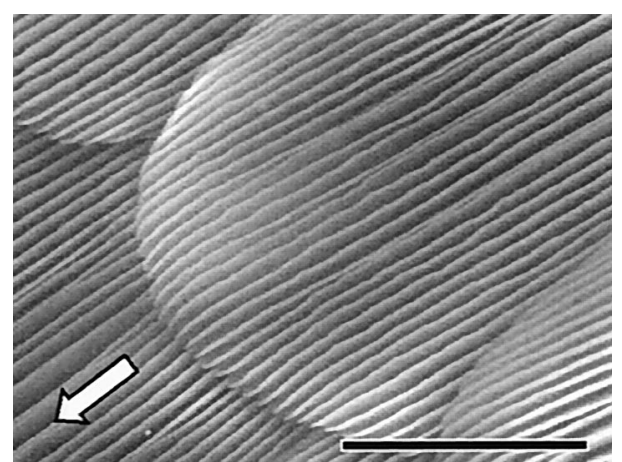

Fig. 3. SEM image of the end of a wing scale, revealing surface ridges. The scale bar represents a distance of $\sim 30 \mu \mathrm{m}$.

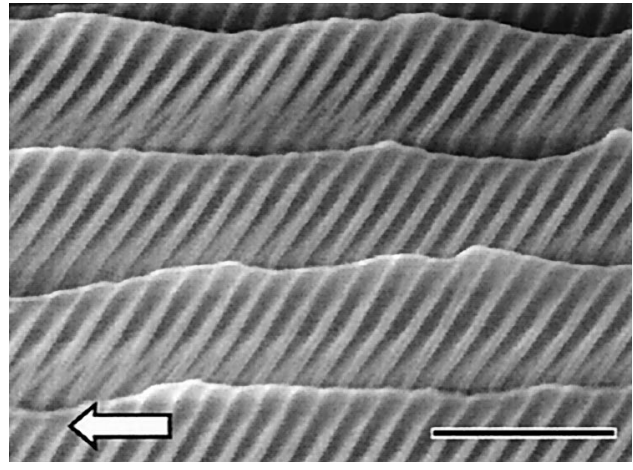

Fig. 4. Side view of ridges presented in Fig. 3, revealing flanges that branch from the ridges. Scale bar is $\sim 1.5 \mu \mathrm{m}$ long.

\section{B. UV-Visible Spectroscopy}

To optically characterize the hind wing it was necessary to obtain measurements of its visible wavelength reflectivity. We achieved this by using a Perkin-Elmer Lambda 900 UV-visible spectrometer with a 150 -mm-diameter integrating sphere attachment, and data were obtained at wavelengths ranging from 380 to $800 \mathrm{~nm}$. The integrating sphere provided a convenient way to characterize the iridescence of the T. magellanus because the sample could be illuminated at a single angle by all wavelengths simultaneously; also the iridescent reflection would be collected no matter what angle it left the wing. (All light that is not absorbed by the wing is reflected around the inside of the sphere until it reaches the exit aperture, from which it is guided to the detector.)

Yellow regions of the hind wings were removed from the two butterflies and mounted onto flat white polyboard substrates. These were placed onto a clamp that was hung in the center of the sphere, and the sample was set at a known angle of incidence $\theta$ (see Fig. 5). The wing was positioned such that the ridges on the scales were parallel to the plane of incidence (to within $\pm 5 \mathrm{deg}$ ). Measurements of the total (specular plus diffuse) reflectivity of the wings were then obtained at values of $\theta$ ranging from the instrumental limits of +60 to $-60 \mathrm{deg}$, these being referenced to a bare polyboard sample at each angle.

Figure 6 presents reflectivity data obtained from
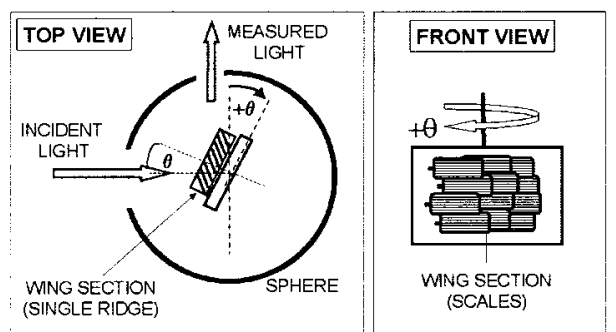

Fig. 5. Schematic of sample orientation in the integrating sphere. Top view illustrates the orientation of multilayer plates with regard to $+\theta$. Incident light that is not absorbed by the sample is reflected from the interior of the sphere until it leaves by a second aperture. The intensity of this light is measured to produce a measure of the total sample reflectivity (specular and diffuse). 


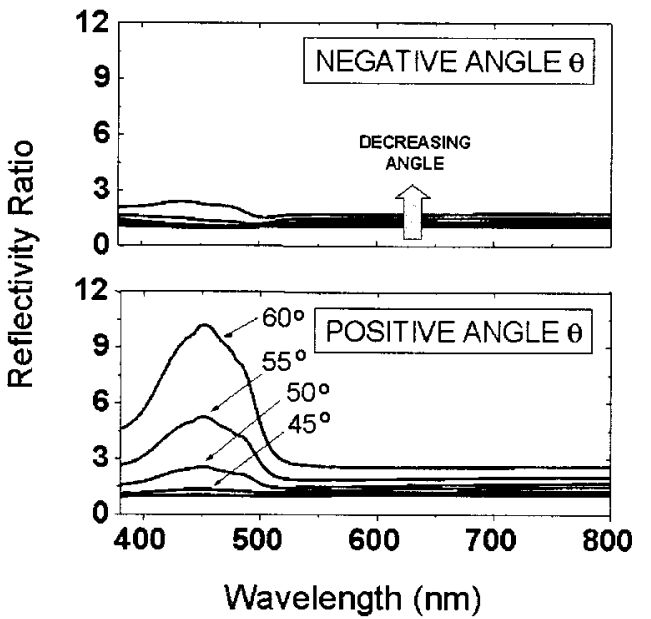

Fig. 6. Reflectivity ratios obtained from the spectrometer. Each data set was normalized to the reflectivity obtained at normal incidence (i.e., $\theta=0$ ).

the T. magellanus sample as the incident angle is varied from -60 to $+60 \mathrm{deg}$. Each data set was ratioed against the normal-incidence $(\theta=0)$ reflectivity of the wing section, and hence the graphs quantify angle-dependent changes in reflectivity. At negative angles there is the expected gradual increase in the reflectivity as grazing incidence is approached, taking the form of a featureless scale-factor increase for angles between 0 and -40 deg. The profile changes slightly for wavelengths below 500 $\mathrm{nm}$ at angles of -50 and $-60 \mathrm{deg}$, but in both cases the reflectivity is comparable to that of higher wavelengths.

At positive angles, however, the situation is different. At angles greater than $+45 \mathrm{deg}$, a reflectivity peak appears, centered at $450 \mathrm{~nm}$ and producing a tenfold increase in intensity at $+60 \mathrm{deg}$ (45\% reflectivity at $450 \mathrm{~nm}$ and $+60 \mathrm{deg}$ ). The iridescence does not exhibit an appreciable peak wavelength shift in either direction as the incident angle is altered and is more intense than would be expected from a multilayer stack that occupies only approximately a third of the scale's surface.

\section{Discussion}

All iridescent effects are necessarily structural in nature, and it is logical to conclude that these platelike projections are the cause of the high incident angle iridescence. However, the flanged ridges are not acting as a simple multilayer reflector.

Instead, the plates present a diffraction grating to the incident light. The termination point of each plate at the top of the ridge acts as a scattering center, forming a 260-nm \pm 5 -nm pitch array in the $x$ direction (see Fig. 7). However, because the plates are tilted there is also a second periodicity with an approximate $360-\mathrm{nm}$ period in the $y$ direction; also, the structure effectively consists of two diffraction gratings: one of several hundred elements parallel

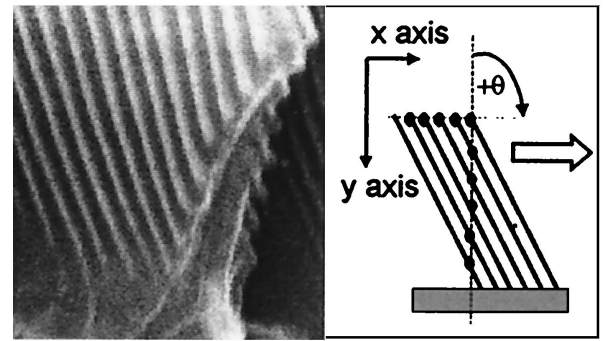

Fig. 7. Definitions of axes and periodicities. The SEM image is of the broken end of a ridge, illustrating the sloping plates. The black circles on the schematic diagram illustrate the two periodic structures that form a lattice of diffracting elements. The white arrow points toward the tip of the hind wing (see Fig. 1).

to the scale's surface and one of only seven elements perpendicular to it.

These two gratings interact, simultaneously diffracting light in the $x$ and $y$ axes. The behavior of such structures, commonly known as bigratings, $, 4,5$ can be predicted when we consider a lattice of discrete points in momentum space (see Fig. 8). The points represent the termination points of momentum vectors that can be applied to incident photons when they are diffracted; hence photon momentum vectors that connect these points are possible solutions to the diffraction equations. The incident and diffracted photon wave vectors must be of the same magnitude (because photon momentum must be conserved) and must join two points on the lattice. Hence, for a photon diffracted from both gratings, there is only one possible photon momentum value $k_{\mathrm{ph}}$ (where $k_{\mathrm{ph}}=2 \pi / \lambda_{\mathrm{ph}}$ and $\lambda_{\mathrm{ph}}$ is the photon wavelength) for a given angle of incidence.

The T. magellanus bigrating is more complex, however, because the grating in the $y$ direction is of limited extent. Diffraction gratings are resonant structures that rely on the interaction of their diffractive elements, and the conditions for resonance become less clearly defined if they consist of fewer than ten periods. ${ }^{6-8}$

A reduction in the number of diffracting elements

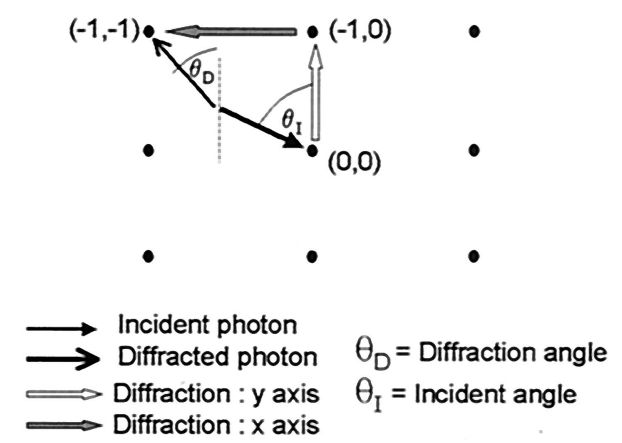

Fig. 8. Momentum-space diagram for infinite lattice of diffractors (i.e., an unrestricted bigrating). Because the photon momentum cannot change during the diffraction process, the incident and diffracted vectors must be of equal length. Hence, for a specific incident angle $\left(\theta_{1}\right)$, only one wavelength can undergo a $(-1,-1)$ scattering process. 


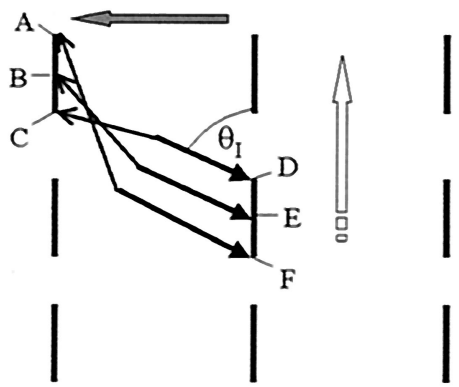

Fig. 9. As per Fig. 8, but for the restricted bigrating of the $T$. magellanus. The points are now elongated in the $y$ direction, and more solutions are possible for a given incident angle. By utilizing different wavelength photons (i.e., vectors of different lengths), we can meet the diffraction conditions by following different paths from one point to another.

in a grating effectively broadens the points into lines (Fig. 9), making new connections possible. Center to center ( $\mathrm{B}$ to $\mathrm{E}$ ) is equivalent to the solutions for the infinite grating case, but paths ranging between $\mathrm{A}$ to $\mathrm{F}$ and $\mathrm{C}$ to $\mathrm{D}$ are also possible, enabling different magnitude vectors of the same incident angle to satisfy diffraction conditions. Hence different wavelength photons that strike the scale at a set angle are all diffracted, but they leave the scale at different angles. This is the phenomenon that produces the butterfly's iridescence.

The extent of this blurring of lattice points can be predicted with Fourier analysis. The scattering intensity from a periodic structure ${ }^{9}$ is proportional to $\phi$ where

$$
\left.\phi=\left\{\sin ^{2}\left[\left(N k_{\mathrm{ph}} d\right) / 2\right]\right\} /\left\{\sin ^{2}\left[k_{p h} d\right) / 2\right]\right\} .
$$

The periodicity is denoted $d$ and $N$ is the number of periods. We used this expression to determine the variation in scattering intensity versus photon momentum for the seven-period grating (Fig. 10). The momentum width of the scattering peak at halfmaximum indicated the extent to which momentumspace lattice points were lengthened (i.e., the lengths of $\mathrm{A}$ to $\mathrm{C}$ and $\mathrm{D}$ to $\mathrm{F}$ in the $y$ direction-see Fig. 9).

Simple geometric arguments now dictate which wavelengths are diffracted for any given angle of incidence. By use of a classical scattering vector notation, ${ }^{10}$ the iridescence is the result of a $(-1,-1)$

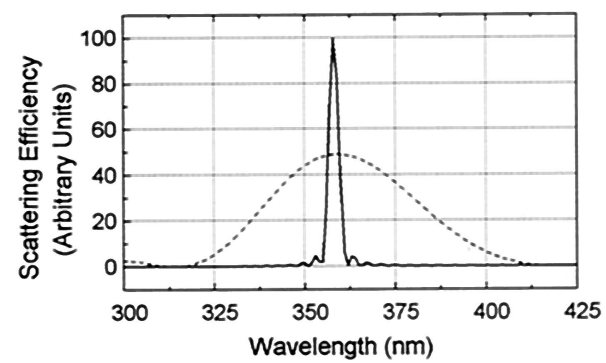

Fig. 10. Calculated relative scattering efficiencies for 100-period (solid curve) and 7-period (dashed curve) gratings. Note the spread in diffracted wavelengths for the restricted grating.

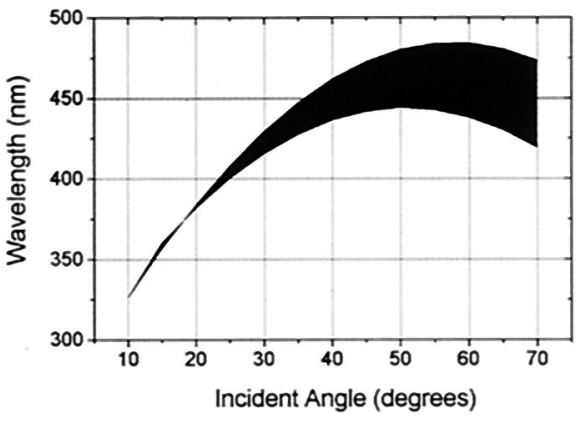

Fig. 11. Prediction of the wavelengths diffracted from the T. $m a-$ gellanus bigrating as a function of incident angle. The shaded region contains the solutions to the diffraction equations; see text for details.

scattering process (i.e., a single backscatter from both individual gratings); the predicted extremes of the wavelength range are plotted in Fig. 11.

Looking at the 40-60 deg region, theory predicts that the range of iridescent wavelengths will extend from approximately 440 to $480 \mathrm{~nm}$. This is a narrower range than is observed experimentally, but its central point is similarly placed and shows the same relative insensitivity to incident angle. Also, at higher angles the iridescence is predicted to become predominantly violet in hue; this could not be measured in the integrating sphere because of experimental limitations, but is in agreement with observations by eye.

\section{Conclusions}

Although many butterflies exhibit iridescence, the optical effect exhibited by the T. magellanus is highly specialized. Previously, constrained bigratings have been detected only in the Ancyluris meliboeus ${ }^{11}$ butterfly, but their effect is different: The multilayer structure is tilted such that the flanges are at $\sim 30$ deg to the substrate, maximizing the angle range over which the iridescence occurs to produce flickering colors. In contrast, the T. magellanus only reveals its structural coloration when both illuminated and viewed at near-grazing incidence and at a specific azimuthal angle. Hence the scale structure dictates both the colors observed and their viewing conditions, producing a unique iridescent effect. Under all other circumstances the appearance of the hind wings is dictated by a highly fluorescent chemical pigment. The $T$. magellanus structure can be thought of as an extreme modification of the A. meliboeus, illustrating the degree of tailoring provided by constrained bigratings.

We thank Andrew Parker (Oxford University) and Helen Ghiradella (State University, New York) for bringing the T. magellanus' iridescence to our attention. This research was carried out as part of Technology Group 08 of the Ministry of Defense Corporate Research Fund and was partly funded by the Biotechnology and Biological Sciences Research Council. 


\section{References}

1. P. Vukusic, J. R. Sambles, and C. R. Lawrence, "Colour mixing in wing scales of a butterfly," Nature (London) 404, 457 (2000).

2. R. B. Morris, "Iridescence from diffraction structures in the wing scales of Callophrys rubi, the Green Hairstreak," J. Entomol. Ser. A 49, 149-154 (1975).

3. P. Vukusic, J. R. Sambles, C. R. Lawrence, and R. J. Wootton, "Quantified interference and diffraction in single Morpho butterfly scales," Proc. R. Soc. London Ser. B 266, 1403-1411 (1999).

4. R. B. Hwang and S. T. Peng, "Performance evaluation of a bigrating as a beam splitter," Appl. Opt. 36, 2011-2018 (1997).

5. J. B. Harris, T. W. Preist, J. R. Sambles, R. N. Thorpe, and R. A. Watts, "Optical response of bigratings," J. Opt. Soc. Am. A 13, 2041-2049 (1996).
6. S. A. Khan, D. N. Qu, and R. E. Burge, "Experimental analysis of diffraction by wavelength-sized metallic gratings in the microwave region," Opt. Eng. 32, 3249-3253 (1993).

7. K. Hirayama, E. N. Glytsis, and T. K. Gaylord, "Rigorous electromagnetic analysis of diffraction by finite-number-of-periods gratings," J. Opt. Soc. Am. A 14, 907-917 (1997).

8. C. R. Lawrence and A. S. Treen, "Polarisation conversion at a textured surface," IEE Proc. Microwave Antennas Propag. 146, 241-246 (1999).

9. M. Born and E. Wolf, Principles of Optics, 4th ed. (Pergamon, New York, 1970), p. 402.

10. N. W. Ashcroft and N. D. Mermin, Solid State Physics (Saunders, Philadelphia, Pa., 1976), Chap. 4.

11. P. Vukusic, J. R. Sambles, C. R. Lawrence, and R. J. Wotton, "Structural colour: now you see it—now you don't," Nature (London) 410, 36-36 (2001). 